\title{
Face Adaptation Effects on Non-Configural Face Information
}

\author{
Ronja Mueller, ${ }^{1,2}$, Sandra Utz ${ }^{3,4,2}$, Claus-Christian Carbon ${ }^{3,4,2}$, and Tilo Strobach ${ }^{1}$ \\ 'Medical School Hamburg, Hamburg, Germany \\ ${ }^{2}$ Bamberg Graduate School of Affective and Cognitive Sciences (BaGrACS), University of Bamberg, Bamberg, Germany \\ ${ }^{3}$ Department of General Psychology and Methodology, University of Bamberg, Bamberg, Germany \\ ${ }^{4}$ Research Group EPAG (Ergonomics, Psychological Æsthetics, Gestalt), Bamberg, Germany
}

ABSTRACT

Inspecting new visual information in a face can affect the perception of subsequently seen faces. In experimental settings for example, previously seen manipulated versions of a face can lead to a clear bias of the participant's perception of subsequent images: Original images are then perceived as manipulated in the opposite direction of the adaptor while images that are more similar to the adaptor are perceived as normal or natural. These so-called face adaptation effects can be a useful tool to provide information about which facial information is processed and stored in facial memory. Most experiments so far used variants of the second-order relationship configural information (e.g., spatial relations between facial features) when investigating these effects. However, non-configural face information (e.g., color) was mainly neglected when focusing on face adaptation, although this type of information plays an important role in face processing. Therefore, we investigated adaptation effects of non-configural face information by employing brightness alterations. Our results provide clear evidence for brightness adaptation effects (Experiment 1). These effects are face-specific to some extent (Experiments 2 and 3) and robust over time (Experiments 4 and 5). They support the assumption that non-configural face information is not only relevant in face perception but also in face retention. Brightness information seems to be stored in memory and thus is even involved in face recognition.

\section{INTRODUCTION}

Despite ongoing changes in our surrounding world, we perceive the environment as rather stable. This impression arises as our cognitive apparatus continuously adjusts to the changing and dynamic attributes of the environment-a process commonly known as figural aftereffect or adaptation effect (Carbon \& Ditye, 2011). Although adaptation occurs largely automatically and usually remains unnoticed, it works effectively in ensuring reliable object recognition. This recognition process is ensured by continuously integrating the adaptation information into the mental object representation stored in memory. However, this integration mechanism also leads to a strong bias in the perception of subsequently inspected stimuli. For example, adapting to a vertical ellipse would cause a circle to appear as slightly elliptical along its horizontal axis (Clifford, 2002; Köhler \& Wallach, 1944;
Suzuki, 2005). Moreover, after inspecting a tilted line, a vertical line would be perceived as slightly tilted in the opposite direction (Gibson, 1933). Thus, the integration of new visual information into the mental representation can lead to a contrastive effect: Subsequent stimuli may be perceived as manipulated in the direction opposite to the adaptor.

Over time, adaptation studies have increasingly focused on more complex objects than simple geometric forms. Many studies investigated adaptation effects on human faces. Human faces are a particularly interesting class of visual information due to their dynamic

Corresponding author: Ronja Mueller, Medical School Hamburg, Department of Psychology, Am Kaiserkai 1, 20457 Hamburg, Germany.

Email: ronja.mueller@medicalschool-hamburg.de 
changes that are based on factors like ageing and different viewing and context conditions. As faces are important for recognizing people and mastering social situations, they seem to be particularly susceptible to adaptation effects that help to update the mental representation. Webster and MacLin (1999) were the first to systematically show visual adaptation effects to alterations in human faces. The authors presented strongly distorted faces in an initial adaptation phase and subsequently observed a strong bias in face perception that was reflected in perceiving the original (i.e., not manipulated) image as being distorted reversely (i.e., in the opposite direction of the adaptor). Since this seminal research, a great number of studies on face adaptation has been performed and various face adaptation effects have been identified. Studies investigating different operational parameters such as the delay between adaptation and test phase revealed that face adaptation effects are highly robust and even transferable to other faces (Carbon \& Ditye, 2011; Carbon et al., 2007). These results indicate that face adaptation effects seem to be better explained by changes on a representational memory level than by changes merely on a perceptual or retinal level. Moreover, the robustness and transferability of the effects (i.e., the sustainability and the transfer to face images other than the adaptor) contradict a simple recency effect (i.e., a tendency to remember the most recently perceived information the best). However, most of the studies investigated adaptation effects on configural face information (i.e., second-order relations; for an overview see Strobach \& Carbon, 2013), although it was hypothesized and also empirically revealed that face recognition is based on further facial information (e.g., Cabeza \& Kato, 2000; Macho \& Leder, 1998; Maurer et al., 2002; Mondloch et al., 2002; Rakover \& Teucher, 1997). This partial neglect of previous studies inspired us to initiate the present study, where we investigated adaptation effects on non-configural face information.

\section{Face Adaptation Effects}

Most face adaptation studies were designed following the same principle: In the initial adaptation phase, a strongly manipulated face is presented. In the subsequent test phase, the participants are asked to choose the veridical (i.e., not manipulated) face out of several face versions displaying the original face along with slightly manipulated versions. The participants usually show a clear bias in their selection toward the previously seen manipulated adaptor. Thus, after adaptation, the original face appears to be manipulated in the opposite direction to the adaptor. Using the example of the so-called face distortion aftereffect, participants would - after seeing a strongly stretched facechoose a slightly stretched face to be the veridical face version since the original version appears compressed (e.g., Carbon \& Ditye, 2011; Carbon \& Leder, 2006; Strobach et al., 2011; Webster \& MacLin, 1999).

This contrasting bias seems to be based on a shift of our mental representation or mental norm of a specific face toward the previously presented adaptor. This shift can be explained by using the face space theory proposed by Valentine (1991). Valentine's theory describes an abstract concept in which mental face representations are located in a multidimensional space. Each face representation reflects the average appearance of a specific individual, built from all encounters one had with this individual. When identifying individuals, their faces are matched against the corresponding mental representations stored in face space. These face representations are located in face space depending on their characteristic value on different feature dimensions (e.g., size of the eyes or shape of the mouth). Face representations that are similar are located very close to each other (since they have similar positions on the feature dimensions), whereas representations that are dissimilar are further apart. This way, a face can be classified along various feature dimensions (Webster \& MacLeod, 2011). Each new experience with a face reshapes the mental representation since new information is immediately integrated into this representation. Hence, an exposure to an extreme alteration in one feature dimension (e.g., an extremely extended face) would cause the mental representation to shift along the specific dimension toward the alteration (see Figure 1 for an illustration). According to Valentine et al. (2016), this mechanism constitutes the adaptation process. When identifying a face (matching the face against the mental representation in face space) after adaptation to a face manipulation, a face image that is similar to

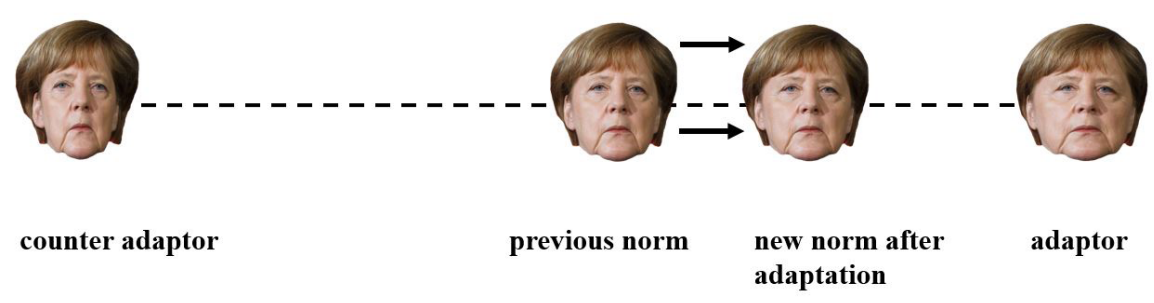

\footnotetext{
FIGURE 1.

A very basic illustration of a representation shift along a feature dimension within the face space (Valentine, 1991). An image of Angela Merkel is used for illustrative purpose. The feature dimension represents a continuum of a specific facial characteristic on which a face can vary (in this case the head's width). The two faces located at the end of the continuum are extreme versions of the feature dimension. The face in the middle represents the initial norm/representation. After adaptation to the right extreme (stretched version of Angela Merkel), the norm is shifted toward the adaptor. The initial norm, however, is perceived as being slightly compressed and appears closer to the left extreme (extremely compressed version). Adapted from Mueller et al. (2020). Permissions and image licenses have been obtained from the copyright holders [Source: @ Drop of Light/Shutterstock.com].
} 
the adaptor would then be perceived as more normal or natural. In contrast, a face image that is similar to the initial mental representation appears as being slightly manipulated in the opposite direction to the adaptor (Mueller et al., 2020).

Adaptation effects have been reported for various types of face information such as ethnicity, identity, gender, expression, or age (Fox \& Barton, 2007; Lai et al., 2012; Leopold et al., 2001; for a review, see Strobach \& Carbon, 2013; Webster et al., 2004). However, these studies investigating face adaptation have one component in common: They used adaptors that were manipulated in terms of configural face information. Configural information refers to the second-order spatial relations between specific facial features (e.g., eye-mouth distance; Maurer et al., 2002). For example, by investigating the aforementioned face distortion aftereffect, Carbon et al. (2007) manipulated the eye-mouth distance and thus created configurally stretched as well as compressed versions of different celebrity faces. A configural manipulation is also performed when investigating face information related to ethnicity, identity, or gender. Studies investigating these types of information often merge different faces or face pairs or create computer generated anti-faces (that reverse the characteristics of the original image, e.g., a female head shape becomes a male head shape, narrow lips become full lips) to generate different levels of ethnicity, identity, gender, and so forth (Fox \& Barton, 2007; Lai et al., 2012; Webster et al., 2004). All these manipulations involve structural and spatial alterations of facial characteristics and thus affect configural aspects.

However, configural face information does not seem to be the only information that is relevant in face processing (although it was most focused on when investigating adaptation effects; see Strobach \& Carbon, 2013, for a review). Non-configural face information that does not affect any relational aspect of the face seems to be involved as well. For example, several studies were able to demonstrate the relevance of feature information (e.g., eyes, nose or mouth; Cabeza \& Kato, 2000; Macho \& Leder, 1998), texture (e.g., Liu et al., 2005; Meinhardt-Injac et al., 2013; O'Neil \& Webster, 2011) or color (e.g., Lee \& Perrett, 1997; Nestor \& Tarr, 2008) in face processing. For instance, Rakover and Teucher (1997) conducted one of the rare studies on the relevance of different facial information by investigating the face inversion effect (i.e., recognition of an inverted face is more difficult than the recognition of an upright face) for either featural or configural face information. They found strong inversion effects for isolated facial features and concluded that these features contribute considerably to face recognition. They assumed that even $91 \%$ of the recognition of an upright human face is actually based on its features.

Liu et al. (2005) presented different grayscale faces that were only defined by texture gradients (the distortion in size or the change in density when objects extend into depth). However, face specific texture information other than texture gradients was no longer accessible since it was manipulated by mapping a fractal-noise pattern onto the face. In a subsequent eight-alternative forced choice test, participants had to identify the previously seen face. The test stimuli were also grayscale faces defined by texture gradients and/or grayscale faces without any fractal-noise patterns that altered the texture of the face. Participants correctly identified the faces above chance but performed poorer than in another experiment where only faces without the fractal-noise pattern were used as targets. The authors concluded that texture gradient information might have been used to recover surface geometry. Thus, texture gradient is probably involved in face processing. Lee and Perrett (1997) investigated the perception of color in faces. They were able to show that participants' accuracy in recognizing celebrity faces was improved when presenting them as caricatures in color space (the results were compared with two other conditions in which either the veridical image or an image with an increased contrast was presented). However, grayscale images decreased participants' accuracy, which indicates that color is an important dimension in facial perception and identification. Taken together, these results clearly show that face perception does not rely solely on configural information, but also involves non-configural face characteristics.

Although many studies show the relevance of non-configural face information in face perception, this type of face information was mainly neglected when investigating adaptation effects. Yamashita et al. (2005) conducted one of the few adaptation studies that implemented an adaptation condition where non-configural face information was used. The authors primarily investigated face adaptation effects to distortions but were also interested in exploring the selectivity of these effects to changes in different facial information. They presented strongly distorted adaptors in the adaptation phase and original and slightly distorted images in the test phase. In addition to the distortions, the authors altered other facial information (configural as well as non-configural information, such as color) of the test images. They found weaker adaptation effects when implementing changes in spatial frequency and contrast polarity (positive vs. negative/inverted contrast) as compared to changes in size, contrast (high vs. low contrast), and color (i.e., red and green chromaticity). Although some dimensions seem to have a greater impact on adaptation effects than others, all facial dimensions actually lead to weaker adaptation effects when altered. This indicates that all implemented dimensions (including non-configural color information) seem to be somehow involved in face processing. However, since these dimensions were only assessed indirectly and in combination with configural distortions, the results cannot clarify whether non-configural face information also contributes to mental representation and long-term retention of faces. Therefore, as to the authors' knowledge, no study directly investigating adaptation effects on non-configural face information exists as of yet.

\section{Processing Levels of Adaptation Effects}

For configural face information, various studies were able to show that adaptation effects are not just processed on a purely sensory level, but they also affect a rather representational memory basis. For example, Carbon et al. (2007) and Carbon and Ditye (2011) showed strong adaptation effects on configurally distorted celebrity faces lasting for one day and even up to one week. These long-term effects cannot be explained by purely perceptual models of adaptation, but indicate sustained modifications of the facial memory. Moreover, they also cannot 
be explained by simple recency effects since recency effects are based on processes of short-term memory and thus would not last a day or even a whole week (especially if distracting stimuli are perceived in the meantime, see e.g., Glanzer \& Cunitz, 1966; Tan \& Ward, 2000). Additionally, in adaptation paradigms where a series of several face versions are presented in the test phase that also includes the strongly manipulated adaptor image, only slightly manipulated face versions are selected (Carbon et al., 2007). This also contradicts a recency effect since a recency effect would lead to a selection of the previous seen adaptor.

Further evidence that adaptation does not operate on a purely sensory processing level is provided by studies investigating different kinds of transfer dimensions. Various authors (e.g, Carbon \& Ditye, 2011, 2012; Carbon et al., 2007; Fox \& Barton, 2007; Strobach et al., 2011; Webster et al., 2004) have found that face adaptation effects not only occur using the identical images as adaptors and test stimuli, but they also transfer across different images of the same identity, as well as across images of different identities (although these effects are usually attenuated). Ghuman et al. (2010) were even able to transfer adaptation effects from bodies onto faces. The authors presented either female or male bodies as adaptors and detected adaptation effects in the perception of gender in subsequent face stimuli. These findings indicate a processing of faces where adaptation affects different hierarchical levels of face representation. Hence, adaptation does not seem to alter only the representation of the presented face, but also representations of specific subpopulations of faces (e.g., faces of the same gender or ethnicity) or a generic norm that represents faces as an object class (Carbon \& Ditye, 2011; Mueller et al., 2020). Thus, durability and transferability seem to be important indicators of the processing level of adaptation effects. Both parameters have been investigated intensively for configural face information but not for non-configural information (e.g., Carbon \& Ditye, 2011, 2012; Carbon \& Leder, 2005; Carbon et al., 2007; Fox \& Barton, 2007; Lai et al., 2012; Otten \& Banaji, 2012; Strobach et al., 2011; Webster et al., 2004). The following study aims to close this gap in the literature.

\section{The Current Study}

Until now, the literature on adaptation appears to have mainly focused on configural face information. In the current study, both the robustness and the transferability of effects were investigated intensively, leading to a relatively profound understanding of how configural face information is represented in memory. However, non-configural face information was mainly neglected when investigating adaptation effects. To the authors' knowledge, previous studies focusing on nonconfigural face information were able to demonstrate the role of nonconfigural information in face perception, but were not able to clarify whether this type of information is also stored in memory. Adaptation paradigms enable the investigation and identification of the information stored in memory. By applying different temporal parameters and transfer levels, the current study investigated the processing level of adaptation effects to non-configural face information. We hereby focused on brightness alterations since it represents more natural changes as compared to, for example, changes in texture or featural information. Moreover, alterations to featural information are confronted with a specific problem: Alterations of features with the methods used so far (i.e., mainly distortions or replacements) also lead to an alteration of configural aspects of the face. By distorting or replacing only one specific unit within a face, the relation to other units is affected too. However, an alteration of facial brightness does not lead to spatial changes and is thus defined as non-configural.

Five experiments are reported in which participants were exposed to celebrity faces that were manipulated in brightness. The manipulation of brightness differed depending on which group the participants belonged to (decreased, no manipulation, or increased brightness). Subsequently, participants were asked to respond to a two-alternative forced choice test in which they were supposed to choose the veridical face out of two alternative faces (the original face and a slightly manipulated version). Following adaptation to strongly manipulated faces, we expected an alteration of the participants' representations of the respective faces toward the adaptor. Thus, after adaptation, the original face would appear manipulated in a direction opposite to the adaptor (e.g., the original would seem to be decreased in brightness after adaptation to a face with increased brightness, and vice versa). Consequently, we expected the participants to select a slightly manipulated face version (e.g., with a slightly increased brightness) to be the veridical face when presented with the two options (original and slightly manipulated face version).

In Experiment 1, we investigated whether face adaptation effects fundamentally exist for non-configural color (i.e., brightness) alterations in celebrity faces. Experiments 2 and 3 attempted to clarify whether possible adaptation effects for brightness alterations are face-specific or whether they also occur to the same extent using non-facial stimuli (or facial stimuli with impaired recognizability, such as inverted faces). Experiments 4 and 5 focused on the durability of possible adaptation effects and thus aimed to determine whether the effects that may have been observed are based on recency effects, addressing a purely sensory level, or if they are based on a representational memory basis (e.g., Carbon \& Ditye, 2011; Carbon et al., 2007). We did so by increasing the interval between adaptation and test stimuli from a relatively short to a longer interval (longer-term adaptation effects are probably processed cognitively higher and might affect the representational memory). In all experiments, we applied different transfer levels (see Figure 2). In the first transfer level (pictorial), the identical images were presented in both the adaptation and test phases. The second transfer level (structural) reflected an experimental condition where the adaptor and test stimulus differ but still represent the same identity (following the study by Carbon \& Leder, 2005 famous faces are used as stimuli). However, in the third transfer level (cross-identity), even different identities were presented in the adaptation and test phase. A comparison of the results of these three conditions can clarify whether the adaptation effects are image- or identity-specific or if they even address higher face concepts (such as the generic norm of faces). 


\section{EXPERIMENT 1}

To find out whether adaptation effects exist for non-configural color information, the first experiment presented celebrity faces manipulated in brightness as adaptation stimuli. In a subsequent test phase, we expected the participants to show a clear bias toward the previously seen manipulated adaptor. The interval between adaptation and test stimuli was 300 $\mathrm{ms}$ and thus rather short.

\section{Method}

\section{PARTICIPANTS}

Forty-eight undergraduate students from the Medical School Hamburg ( 47 females, 1 male, $M_{\text {age }}=22.7$ years, range $=18$ to 44 years) were tested individually. The needed minimal sample size of $N=45$ was calculated a priori via power analysis (Faul et al., 2007) based on a mixed-design analysis of variance (ANOVA) with a 3 (between-subjects) $\times 3$ (within-subjects) factor design being able to detect a mediumlarge effect size $\mathrm{f}$ of 0.25 (Cohen, 1988) given an $\alpha=.05$ and a test power $(1-\beta)=0.90$. The effective $N$ was 48 , resulting in an increased test power of 0.93 . Study participation was rewarded with either money $(€ 12)$ or credit points (as part of course requirements). All participants were naïve to the purpose of the experiment. To test for anomalies in color perception, we employed a short version of the Ishihara color test (Ishihara, 1917). Vision was further assessed with the Freiburg Visual Acuity and Contrast Test (FrACT; Bach, 1996). Only participants with normal or corrected-to-normal vision were included in the subsequent testing. The participants were randomly assigned to one of the three participant groups (adaptation stimuli decreased in brightness, original images, or stimuli increased in brightness). The study was conducted according to the guidelines of the Declaration of Helsinki. All participants provided written informed consent. The study was approved by the ethics board of the Medical School Hamburg (date of application: March 09, 2017).

\section{APPARATUS AND STIMULI}

A list of 70 celebrity names was generated by studying articles of tabloid journalism and interviewing colleagues. A color photograph of each celebrity from the list was collected and displayed in a subsequent survey in which we asked the participants (92 participants; 66 females, 26 males, $M_{\text {age }}=21.7$ years, range $=18$ to 30 years) to write down the name of the celebrities (as far as they knew it) and judge their familiarity on a 5 -point Likert scale ( $1=$ unfamiliar; $5=$ very familiar $)$. People who participated in the survey were excluded from further experiments. On the basis of the survey data, we selected 30 celebrities $^{1}$ who were named most frequently and rated highest in terms of familiarity. Two color photographs (A and B) of each of these 30 "most famous" celebrities were collected and selected according to the following criteria: The photographs should present a full face in a frontal view, in high resolution, and with a straight gaze. Furthermore, it was ensured that hair did not cover any facial features (such as eyes, nose, or mouth) and that celebrities did not wear glasses. These images served as the basic stimulus material for the conducted experiments. All selected celebrity photos were randomly divided into three groups. Since there were two photos of each celebrity (A and B), there were a total of six different stimulus sets. These sets served to investigate the possible transfer of adaptation effects by forming the three different transfer levels that varied in terms of the overlapping information between images seen in the adaptation and test phase. On the first transfer level (pictorial), the stimuli of the adaptation and test phase were pictorially identical (e.g., presenting Image A of George Clooney as an adaptor and the identical image as the test stimulus). On the second transfer level (structural), the adaptation and test image differed from each other but still showed the same identity (e.g., displaying Image A of Heidi Klum as an adaptor and Image B of Heidi Klum as a test stimulus). On the third transfer level (cross-identity), different identities were shown in the adaptation and test phases (e.g., presenting Image A of Angela Merkel as an adaptor and Image A of Brad Pitt as test stimuli). The three different transfer levels that were employed as a within-subjects factor are illustrated in Figure 2.

The brightness of all images was manipulated (using Adobe Photoshop CC, Version 19.0), resulting in five different image versions, as illustrated in Figure 3. Either the original image (ORIGINAL, representing the control group), an image with a strongly decreased brightness (-75\%, MINUS EXTREME), or an image with a strongly increased brightness (+75\%, PLUS EXTREME) were presented as adaptors, depending on which adaptation group the participant belonged to (between-subjects factor). The adaptation images (PLUS EXTREME and MINUS EXTREME) were to be clearly recognized as manipulations.

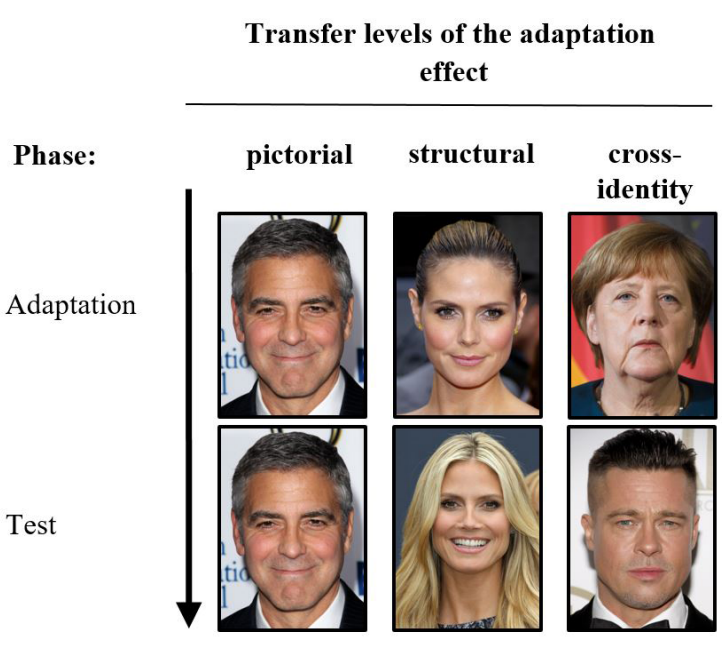

\section{FIGURE 2.}

Illustration of the different transfer levels. Each column represents one trial with a different transfer condition being applied. The left column represents a trial of the pictorial transfer level, the column in the middle represents a trial of the structural transfer level, and the right column represents a trial of the cross-identity transfer level. The presented images are used for illustrative purpose only and were not used in the original study. Permissions and image licenses have been obtained from the copyright holders [Sources: (c) Drop of Light/Shutterstock.com, Tinseltown/Shutterstock.com, s_bukley/Shutterstock.com] 


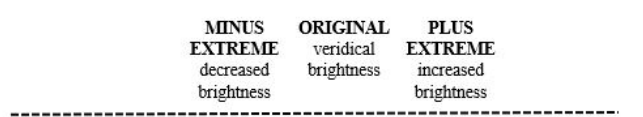

Exp. 1, 4, 5:
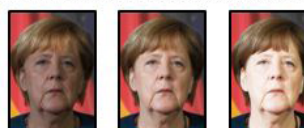

A

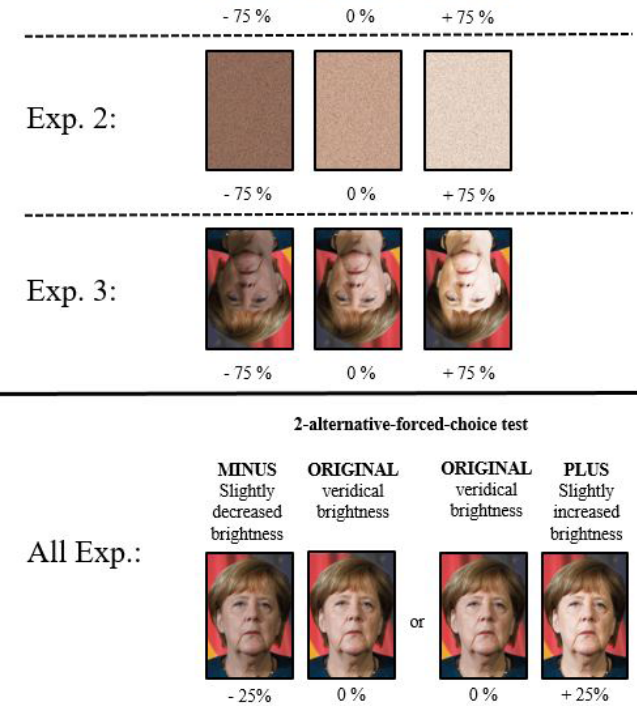

\section{FIGURE 3.}

Illustration of the different image versions. Panel (A): Illustration of the different image versions during the adaptation phase. Experiments 1, 4 and 5 used the same stimulus material in the adaptation phase. Experiment 2 displays scrambled face stimuli, and Experiment 3 displays inverted face stimuli as adaptors. Panel (B): Illustration of the different image versions within the test phase. Within the two-alternative-forced-choice test, two images are presented (either an image with decreased brightness or an image with increased brightness, together with the original image). All experiments use the same stimulus material in the test phase. Angela Merkel is used here as an example. Permissions and image licenses have been obtained from the copyright holders [Sources: @ Drop of Light/Shutterstock.com].

The test stimuli were the ORIGINAL images and a version with either a slightly decreased brightness (-25\%, MINUS) or slightly increased brightness ( $+25 \%$, PLUS). Since the images could have already been, for example, manipulated by the photographer, it cannot be guaranteed that the images were completely unedited when selected. Consequently, the term "ORIGINAL image" should be used with caution, since it might not represent the original (unedited) identity. Instead, the term refers mainly to the fact that the ORIGINAL image was not manipulated in brightness. Moreover, since the selected images showed no obvious prior manipulations, they should be able to sufficiently reflect the accumulated experience with the depicted identities. The size of the images for adaptation and test phase was approximately $330 \times 412$ pixels. The experiment was created with Experiment Builder 2.2.1 (SR Research) and ran on a Lenovo PC with a 23-inch monitor and a resolution of $1920 \times 1080$ pixels.

\section{PROCEDURE}

Experiment 1 presented the adaptation and test phase within one trial, separated by a $300 \mathrm{~ms}$ interstimulus interval. As illustrated in Figure 4, each trial started with a fixation cross (displayed for $500 \mathrm{~ms}$ and placed in the center of the subsequent stimulus position) before presenting the adaptor. Depending on which group they belonged to, participants then either inspected an ORIGINAL image or one of the two extreme versions of this image (MINUS EXTREME or PLUS EXTREME, see Figure 3 for an illustration). In each participant group, all 30 celebrity images were presented. Moreover, the celebrity images were displayed with the same frequency. To increase the inspection time of the adaptation stimuli while avoiding fatigue effects, the variability of the task was increased by presenting the adaptors for either 2, 3 or $4 \mathrm{~s}-$ a technique that was already successfully employed by Carbon et al. (2007). To control for retinal effects, the adaptor was placed in one of six different screen positions (top-left, top-center, top-right, bottom-left, bottom-center, or bottom-right). The different presentation times and screen positions of the adaptor were balanced throughout the experiment. Each adaptor was shown twice on each screen position and four times at each presentation time. Hence, a single adaptor was shown 12 times (i.e., in 12 different trials) during the whole experiment. Participants adapted to two sets of celebrity faces (i.e., one image set [A or B] of two [out of three] different celebrity groups). Celebrities assigned to the third group were not shown during adaptation. The image sets that were used for the adaptation phase (and also their order) were determined in advance for each participant and balanced across participants throughout the entire experiment. After the exposure to the adaptor, a backward mask appeared in order to eliminate possible afterimages (Turvey, 1973) and a blank screen was presented. The mask and the blank screen were shown for $150 \mathrm{~ms}$ each, resulting in an interstimulus interval (between adaptor and test stimuli) of $300 \mathrm{~ms}$.

After the blank screen, a two-alternative forced-choice test was presented, showing two different image versions of the same picture: either a MINUS image ( $-25 \%$ brightness) or a PLUS image ( $+25 \%$ brightness), together with an ORIGINAL image. Stimulus position was randomized and balanced across trials (ORIGINAL left/MINUS right; MINUS left/ORIGINAL right; ORIGINAL left/PLUS right; PLUS left/ORIGINAL right). While in the adaptation phase only two sets of celebrity faces were presented, three sets were displayed in the test phase, belonging to the three celebrity groups. To apply the three different transfer levels, either the same or the corresponding image set (A or B) of each celebrity group (1,2, or 3 ) was presented, depending on the image sets presented in the adaptation phase. For the first transfer level (pictorial), the image set was identical to the first image set of the adaptation phase (e.g., presenting Image Set A of Celebrity Group 1 in both the adaptation and test phase). For the second transfer level (structural), the second image set of the corresponding celebrity group was presented (e.g., presenting Image Set A of Celebrity Group 2 in the adaptation phase and Image Set B of the same celebrity group in the test phase). To apply the third transfer level (cross-identity), images of the celebrity group that was not shown during adaptation were 
used as test targets, and images presented in the adaptation phase of the other transfer conditions were used as adaptors (e.g., presenting Image Set A of Celebrity Group 1 in the adaptation phase and Image Set A of Group 3 in the test phase). The two adaptor sets that were used to apply the pictorial and structural transfer level (e.g., Image Sets $1 \mathrm{~A}$ and $2 \mathrm{~A}$ ) also served to apply the cross-identity transfer level. Half of the image set that served to apply the pictorial transfer level (e.g., 15 out of 30 images of Image Set 1A) and half of the image set that served to apply the structural level (e.g., 15 out of 30 images of Image Set 2A) was used for the cross-identity transfer level (e.g., half of the Image Set $1 \mathrm{~A}$ and half of the Image Set $2 \mathrm{~A}$ were presented in the adaptation phase and Image Set $3 \mathrm{~A}$ was displayed in the test phase). The other halves of the image sets used for the pictorial and structural transfer level were not presented in the cross-identity transfer level (i.e., they were not presented a second time). Hence, each adaptor was either presented just in one or two (either pictorial, pictorial and cross-identity, structural or structural and cross-identity) transfer conditions. This imbalance was corrected by balancing the frequency of adaptor presentation across the experiment.

The image sets used for the test phase were balanced across participants throughout the entire experiment. Within each participant group and across all three transfer levels, the brightness of the adaptors was kept constant. While the selection of the presented image sets was determined in advance for each participant, the trial and stimulus order were randomized. In each trial, the test images were presented for $1.500 \mathrm{~ms}$. Backward masks appeared (for $300 \mathrm{~ms}$ ) after each test-stimulus presentation in order to avoid afterimages and thus an extended exposure to the test stimuli. After the masking, participants were asked to select the veridical image (i.e., the image that most accurately represents the identity) from the two previously presented images. Instructions were: "Which is the original face?" (in German). The selection was indicated by pressing a specific button on a keyboard ("A" and "L"). Participants were explicitly instructed at the beginning of the experiment to base their selection on the memory about the celebrity (e.g., images seen in the media) and not on what they had experienced within the experiment. This way, participants were encouraged to access the representation stored in memory when making their selection. Since the adaptation images were obviously manipulated and thus did not represent the original identity, it was clear to the participants that they were not supposed to base their response on the adaptors seen before. Altogether, the experiment included 360 trials and lasted about 40 minutes. Halfway through the experiment (i.e., after 180 trials), participants were presented with a text informing them that they had completed the first half of the experiment. Participants were allowed to take a short break and were free to decide when to start the second half of the experiment.

After the adaptation and test phases, participants were given the task of judging the celebrities to which they were exposed according to their familiarity (whether they have seen the celebrities before in the media). Instructions regarding the familiarity were: "Are you familiar with this celebrity from the media?" (in German). Participants responded with either "yes" (“A”) or "no" (“L”). The aim of this task was to ensure that the participants based their selection about the veridicality of the identities in the task before (i.e., the test phase of

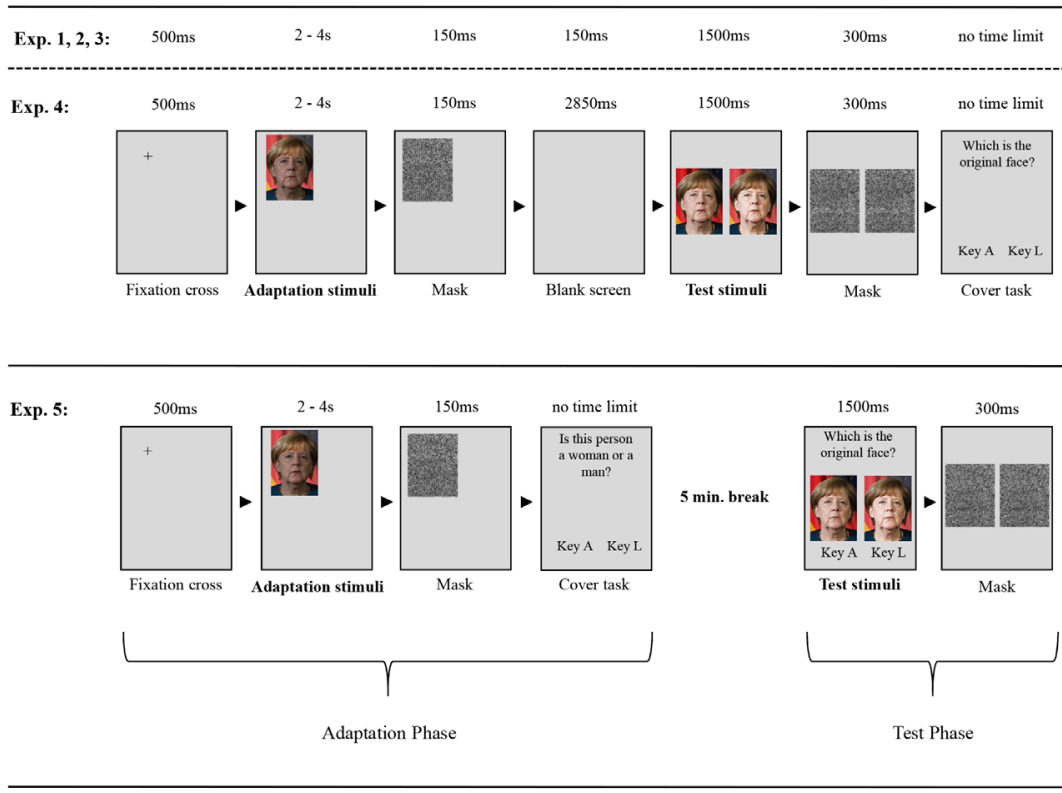

\section{FIGURE 4.}

Schematic illustration of the trial structure of Experiments 1 to 5 . Experiments 1 to 3 are similar in their timing (but differ in their stimulus material, see Figure 3). Experiments 1 to 3 and Experiment 4 differ only in their interstimulus interval (a blank screen is presented either for 150 ms or 2850 ms, resulting in an interstimulus interval of either 300 ms or 3000 ms). In Experiment 5, the adaptation and test phases are presented separately (with a 5 min. break in between). An image of Angela Merkel is used here as an example. Permissions and image licenses have been obtained from the copyright holders [Sources: @ Drop of Light/Shutterstock.com]. 
the adaptation paradigm) on an internal mental representation. Since the memory probably contains stable mental representations of only familiar identities, the rating was used to exclude trials displaying celebrities that were unfamiliar to the participants.

\section{Results and Discussion}

On average, $96.7 \%$ of the celebrity faces presented in the experiment were considered to be familiar (individually ranging from $73.3 \%$ to $100 \%)$. Trials that displayed a celebrity who was not recognized by the participant were excluded from further analysis. Furthermore, all trials with a reaction time (RT) faster than $200 \mathrm{~ms}$, as well as all individual outliers (i.e., RT slower than 3 SDs above the individual mean RT) of each participant, were excluded. The average selection of test faces for each participant was used as the dependent variable (later referred to as target selection). The selection was scored according to the alteration of the selected image: A score of -25 was used for MINUS images; a score of 0 was used for ORIGINAL images; and a score of +25 was used for PLUS images ${ }^{2}$.

A two-way, mixed-design analysis of variance (ANOVA) was conducted with the between-subjects factor of adaptation group (MINUS EXTREME, ORIGINAL, and PLUS EXTREME) and the within-subjects factor of transfer level (pictorial, structural, and crossidentity). There was a significant main effect of adaptation group, $F(2$, $45)=13.781, p<.001, \mathrm{n}_{\mathrm{p}}{ }^{2}=.380$ (MINUS EXTREME: $M=-8.84$, $S D=2.58$; ORIGINAL: $M=-3.78, S D=4.87$; PLUS EXTREME: $M=$ $1.12, S D=7.51)$. Bonferroni adjusted comparisons revealed significant differences between all possible pairs (between MINUS EXTREME and ORIGINAL: $p=.032$; between ORIGINAL and PLUS EXTREME: $p=.040$; and between MINUS EXTREME and PLUS EXTREME: $p$ $<.001)$. Since the results revealed the expected pattern in the participants' target selection (i.e., bias of the participants' selection toward images with a reduced brightness, i.e., numerically small values, after inspecting images with a strongly reduced brightness, and a contrasting bias, i.e., numerically higher values, after inspecting images with a strongly increased brightness), a general adaptation effect on brightness is indicated. There was no main effect of transfer level, $F(2,90)<1$, $p=.581$, but there was a significant interaction of adaptation group and transfer level, $F(4,90)=4.102, p=.004, \mathrm{n}_{\mathrm{p}}{ }^{2}=.154$, see Figure 5A for an illustration. Hence, the transfer levels differed statistically significantly in their average values (reflecting the average selection of the test faces). Moreover, these significant differences between transfer levels seemed to vary within a different range for each participant group.

Univariate ANOVAs of the factor adaptation group showed large effects for all transfer levels: pictorial, $F(2,45)=18.873, p<.001, \mathrm{n}_{\mathrm{p}}{ }^{2}$ $=.456$; structural, $F(2,45)=10.589, p<.001, \mathrm{n}_{\mathrm{p}}{ }^{2}=.320$; and crossidentity, $F(2,45)=9.138, p<.001, \mathrm{n}_{\mathrm{p}}{ }^{2}=.288$. Within the MINUS EXTREME adaptation group, a greater effect was observed for the pictorial compared to the cross-identity transfer level, $p=.013$. There was no difference between the pictorial and structural, nor between the structural and cross-identity transfer levels, $p s>.188$. Within the PLUS EXTREME adaptation group, a greater effect was observed for the pictorial compared to the cross-identity transfer level, $p=.001$, and also for the pictorial compared to the structural transfer level, $p=$ .048. There was no difference between the structural and cross-identity transfer levels, $p=.409$. As expected, the control group (ORIGINAL) did not show any differences between the transfer levels, $p s>.153$. Thus, it seems that although all transfer levels showed a large adaptation effect across the experimental groups, the pictorial transfer level showed the largest effect. This indicates that the observed adaptation effect operates to a certain extent only on an image-specific level. However, the effect is also transferable to other images or even to other identities, although it is attenuated. Thus, faces strongly altered in brightness can bias the subsequent perception of identical images, different image versions, and also the perception of other identities. Since Figure 5A indicates that there could be a shift in the average selection of all participant groups toward the negative pole, a one-sample $t$-test was conducted comparing the average selection across all groups. Since we expected a value around zero for the average selection of the control group and positive or negative values of similar magnitude for the two adaptation groups, a total average value (across all groups) around zero would be anticipated (as the values of the adaptation groups would abrogate each other). Thus, a significant negative deviation from zero would indicate a bias of the overall results into the negative range. The one-sample $t$-test indeed showed a negative bias: $M=-3.83 ; t(47)=$
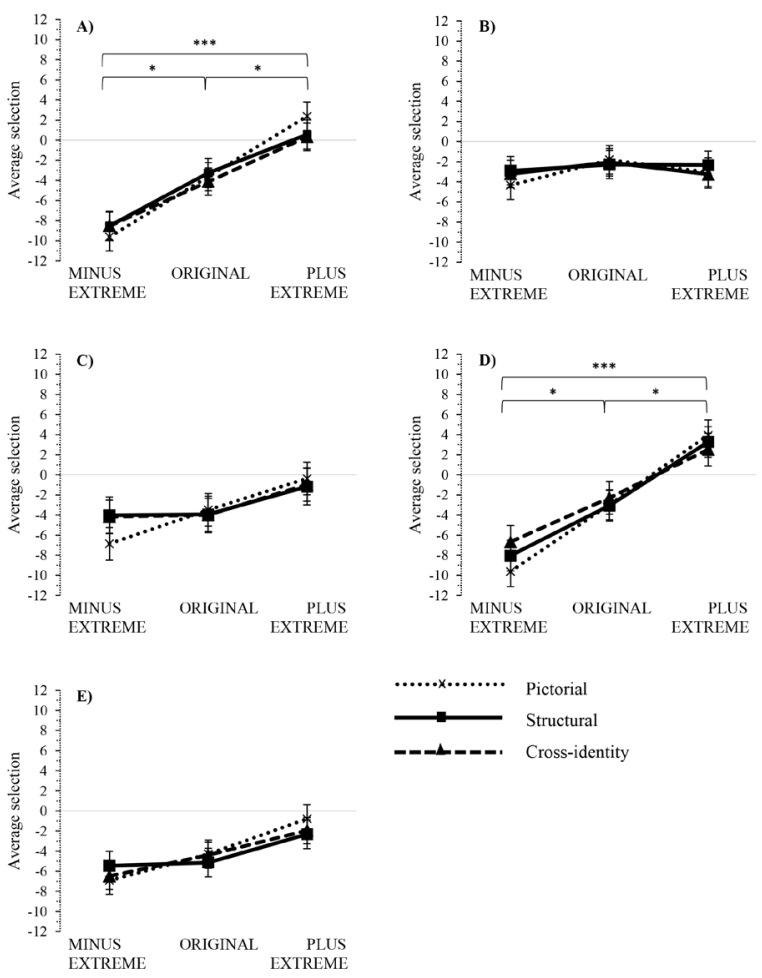

\section{FIGURE 5.}

Illustration of the interaction between adaptation group and transfer level for all experiments. Panel (A): Experiment 1. Panel (B): Experiment 2. Panel (C): Experiment 3. Panel (D): Experiment 4. Panel (E): Experiment 5. The "average selection" axis represents the mean brightness of the selected test stimuli (in \%). Error bars represent \pm 1 standard error of the mean. 
$-3.981 ; p<.001$ (one-tailed); $d=-0.575$. Thus, overall, the participants selected images with a lower brightness than expected.

\section{EXPERIMENT 2}

Since the results of Experiment 1 suggested that face adaptation effects also exist for non-configural color (i.e., brightness) information, we wanted to clarify in the following experiment whether the detected brightness adaptation effects are selective for faces or whether they are instead general color aftereffects that can be provoked by other stimulus material than faces. Therefore, Experiment 2 used non-face stimuli in the adaptation phase that were manipulated in brightness. However, test stimuli did not differ from Experiment 1 and thus presented celebrity faces. The non-face adaptation stimuli were created by scrambling beyond recognition the faces initially presented in the adaptation phase in Experiment 1 (see Figure 3A). Each image was divided into very small pieces and randomly assembled so that one homogeneous color area was created, representing the average color of the respective adaptation image from Experiment 1 (a so-called scrambled face). Since the scrambled-face stimuli did not display any face, adaptation effects in this experiment should be reduced compared to the effects of Experiment 1 if the reported adaptation effects of Experiment 1 are indeed face-specific. As the adaptor stimuli no longer displayed the celebrities presented in the test phase, the pictorial and structural transfer levels no longer applied. Nevertheless, for the sake of consistency between experiments, the conditions shall be listed in this experiment.

\section{Method}

\section{PARTICIPANTS}

Forty-eight undergraduate students from the University of Bamberg (38 females, 10 males, $M_{\text {age }}=24.5$ years, range $=19$ to 41 years) were tested individually. The sample size was the same as in Experiment 1. Study participation was rewarded with either money $(€ 12)$ or credit points (as part of course requirements). All study requirements and conditions were the same as in Experiment 1. None of the participants had taken part in Experiment 1.

\section{APPARATUS, STIMULI, AND PROCEDURE}

Experiment 2 was identical to Experiment 1 except the following: The adaptation stimuli (but not the test stimuli) used in this experiment were scrambled versions of the stimuli presented in Experiment 1 (see Figure 3 ). The images were scrambled in such a way that face recognition was no longer possible.

\section{RESULTS AND DISCUSSION}

On average, $94.9 \%$ of the celebrity faces presented in the experiment were considered to be familiar (individually ranging from $46.7 \%$ to $100 \%)$. As in Experiment 1, all trials that displayed a celebrity who was not recognized by the participant were excluded from further analysis, as well as trials with an RT faster than $200 \mathrm{~ms}$ and the individual outliers (i.e., RT slower than 3 SDs above the mean of each participant).
The target selection was used as the dependent variable. The selection was scored according to the alteration of the selected image (a score of -25 was used for MINUS images; 0 for ORIGINAL images; and +25 for PLUS images).

A two-way, mixed-design ANOVA was conducted with the between-subjects factor of adaptation group (MINUS EXTREME, ORIGINAL, and PLUS EXTREME) and the within-subjects factor of transfer level (pictorial, structural, and cross-identity). There was no significant main effect of adaptation group, $F(2,45)<1, p=.730$ (MINUS EXTREME: $M=-3.48, S D=5.48$; ORIGINAL: $M=-2.06$, $S D=4.47$; and PLUS EXTREME: $M=-2.88, S D=5.18$ ). Furthermore, there was neither a main effect for transfer level, $F(2,90)<1, p=.619$, nor an interaction between adaptation group and transfer level, $F(4$, $90)<1, p=.633$, see Figure 5B for an illustration. Thus, we were not able to detect an adaptation effect regarding brightness alterations of scrambled faces in Experiment 2. Although no adaptation effect was observed, the graph indicates that there could have been a shift in the average selection of all participant groups into the negative range (see Figure 5B). A one sample $t$-test indeed revealed a significant negative bias of the overall results, $M=-2.83 ; t(47)=-3.927 ; p<.001$ (onetailed); $d=-0.567$. Thus, although no adaptation effects were revealed, the participants showed a tendency of selecting celebrity faces decreased in brightness as the veridical images.

\section{EXPERIMENT 3}

Since the scrambled faces differed significantly in contrast and complexity in comparison to the adaptation images presented in Experiment 1, the results of Experiment 2 cannot completely clarify whether the results obtained from Experiment 1 are face-specific or not. The absence of adaptation effects could just as well be explained by the great difference of the adaptation and test stimuli. Thus, the following experiment was carried out using adaptation images that were more similar (e.g., in color composition and complexity) to the adaptor images used in Experiment 1 and to the test images. Experiment 3 used the adaptation images of Experiment 1 as inverted images (see Figure 3A). Inverted face stimuli do not differ from upright images except in orientation. Hence, they are much more comparable to the images of Experiment 1 than the scrambled face stimuli implemented in Experiment 2. Moreover, inverted face stimuli are even more comparable than, for example, images of other object classes (e.g., houses, cars, etc.), since they still display the same faces as used in Experiment 1. Nevertheless, face inversion causes strong recognition impairments and thus can serve as a kind of "non-face stimulus" (for a review see Valentine, 1988; e.g., Yin, 1969). If adaptation effects are revealed in Experiment 3, the results of Experiment 1 can be interpreted as being specific for upright faces. As the adaptor stimuli differed from the test images in their orientation, the use of the term "pictorial" might be misleading. Nevertheless, the term will be used for the sake of consistency with the descriptions of the other experiments. However, in the context of Experiment 3, the term "pictorial" means the pictorial congruence of the basic image, without inversion or brightness alterations 


\section{Method}

\section{PARTICIPANTS}

Forty-eight undergraduate students from the Medical School Hamburg ( 33 females, 15 males, $M_{\text {age }}=23.0$ years, range $=18$ to 31 years) were tested individually. The sample size was the same as in Experiments 1 and 2. Study participation was rewarded with either money (€12) or credit points (as part of course requirements). All study requirements and conditions were the same as in the previous experiments. None of the participants had taken part in Experiments 1 or 2.

\section{APPARATUS, STIMULI, AND PROCEDURE}

This experiment was identical to Experiment 1 except the following: The adaptation stimuli (but not the test stimuli) used in this experiment were inverted versions of the stimuli presented in Experiment 1 (see Figure 3).

\section{RESULTS AND DISCUSSION}

On average, $97.3 \%$ of the celebrity faces presented in the experiment were considered to be familiar (individually ranging from $63.3 \%$ to $100 \%)$. As in Experiments 1 and 2, all trials that displayed a celebrity who was not recognized by the participant were excluded from further analysis, as well as trials with an RT faster than $200 \mathrm{~ms}$ and the individual outliers (i.e., RT slower than $3 S D$ s above the mean of each participant). The target selection was used as the dependent variable. The selection was scored according to the alteration of the selected image (a score of -25 was used for MINUS images; 0 for ORIGINAL images; and +25 for PLUS images).

A two-way, mixed-design ANOVA was conducted with the between-subjects factor of adaptation group (MINUS EXTREME, ORIGINAL, and PLUS EXTREME) and the within-subjects factor of transfer level (pictorial, structural, and cross-identity). There was no significant main effect of adaptation group, $F(2,45)=1.182, p=.316$ (MINUS EXTREME: $M=-4.72, S D=4.96$; ORIGINAL: $M=-4.46$, $S D=4.40$; and PLUS EXTREME: $M=-1.96, S D=7.15)$. Furthermore, there was no main effect of transfer level, $F(2,90)<1, p=.689$, nor an interaction of adaptation group and transfer level, $F(4,90)=1.863, p=$ .124 (see Figure 5C for an illustration). Thus, we were not able to detect an adaptation effect regarding brightness alterations of inverted faces in Experiment 3. As in Experiment 2, the graph indicates that there could have been a shift in the average selection of all participant groups into the negative range (see Figure 5C). A one-sample $t$-test indeed revealed a significant negative bias of the overall results, $M=-3.74 ; t(47)=$ $-4.585 ; p<.001$ (one-tailed); $d=-0.662$. Thus, although no adaptation effects were revealed, the participants showed a tendency of selecting celebrity faces decreased in brightness as the veridical images.

\section{EXPERIMENT 4}

Experiments 1, 2, and 3 were able to demonstrate that face adaptation effects also exist for non-configural color (i.e., brightness) information and that these effects are somehow face-specific. In the following experiments, we wanted to clarify the processing level of the adaptation effects. Since temporal factors of the adaptation paradigm seem to be a key variable for investigating the level of face processing, timing was relevant in the following two experiments. An extension of the interstimulus interval enables an identification of sensory-based (retinotopic) effects versus more robust and thus cognitively higher processed effects. Experiment 4 only slightly increased the interstimulus interval from milliseconds (300 ms in Experiments 1 to 3) to seconds $(3 \mathrm{~s})$.

\section{Method}

\section{PARTICIPANTS}

Forty-eight undergraduate students from the Medical School Hamburg ( 39 females, 9 males, $M_{\text {age }}=23.9$ years, range $=19$ to 31 years) were tested individually. The sample size as well as all study requirements and conditions were the same as in the previous experiments. None of the participants had taken part in the previous experiments.

\section{APPARATUS AND STIMULI}

The apparatus and stimuli were the same as those used in Experiment 1 (see Figure 3).

\section{PROCEDURE}

The procedure was identical to that of Experiment 1, except that the interstimulus interval was extended to $3 \mathrm{~s}$. Thus, following the adaptor and preceding the presentation of the test stimuli, a backward mask appeared for $150 \mathrm{~ms}$, and a subsequent blank screen appeared for $2850 \mathrm{~ms}$ (see Figure 4).

\section{RESULTS AND DISCUSSION}

All trials with an RT faster than 200 ms were excluded, as well as all individual outliers (i.e., RT slower than $3 S D$ s above the mean of each participant). The target selection was used as the dependent variable. The selection was scored according to the alteration of the selected image (a score of -25 was used for MINUS images; 0 for ORIGINAL images; and +25 for PLUS images). Besides this outlier analysis, we included all trials in this experiment. A two-way, mixeddesign ANOVA was conducted with the between-subjects factor of adaptation group (MINUS EXTREME, ORIGINAL, and PLUS EXTREME) and the within-subjects factor of transfer level (pictorial, structural, and cross-identity). There was a significant main effect of adaptation group, $F(2,45)=14.539, p<.001, \mathrm{n}_{\mathrm{p}}{ }^{2}=.393$ (MINUS EXTREME: $M=-8.10, S D=3.77$; ORIGINAL: $M=-2.77$, $S D=4.90$; and PLUS EXTREME: $M=3.25, S D=8.27$ ). Bonferroni adjusted comparisons revealed significant differences between all possible pairs (between MINUS EXTREME and ORIGINAL: $p=$ .045 ; between ORIGINAL and PLUS EXTREME: $p=.019$; and between MINUS EXTREME and PLUS EXTREME: $p<.001$ ). Since the results revealed the expected pattern in the participants' target 
selection (i.e., bias of the participants' selection toward images with a reduced brightness after inspecting images with a strongly reduced brightness, and a contrasting bias after inspecting images with a strongly increased brightness), a general adaptation effect on brightness is indicated. There was no main effect of transfer level, $F(2,90)$ $=1.460, p=.238$, but there was a significant interaction of adaptation group and transfer level, $F(4,90)=4.351, p=.003, \mathrm{n}_{\mathrm{p}}{ }^{2}=.162$ (see Figure 5D for an illustration).

Univariate ANOVAs of adaptation group showed large effects for all transfer levels: pictorial, $F(2,45)=20.488, p<.001, \mathrm{n}_{\mathrm{p}}{ }^{2}=.477$; structural, $F(2,45)=13.902, p<.001, \mathrm{n}_{\mathrm{p}}{ }^{2}=.381$; and cross-identity, $F(2,45)=7.929, p=.001, \mathrm{n}_{\mathrm{p}}{ }^{2}=.261$. Within the MINUS EXTREME adaptation group, a greater effect was observed for the pictorial compared to the cross-identity transfer level, $p<.001$. There was also a significant difference between the pictorial and structural transfer levels, $p=.050$, but not between the structural and cross-identity transfer levels, $p=.096$. Within the PLUS EXTREME adaptation group, a greater effect was observed for the pictorial compared to the cross-identity transfer level, $p=.034$. There was no significant difference between the pictorial and structural transfer levels, $p=$ .386, nor between the structural and cross-identity transfer levels, $p$ $=.357$. As expected, the control group (ORIGINAL) did not show any differences between the transfer levels, $p s>.303$. Thus, we observed the same pattern as in Experiment 1: Although all transfer conditions showed a large adaptation effect across the experimental groups, the pictorial transfer condition showed the largest effects, indicating an image-specific component within the adaptation effect. However, since the effect was also transferable to other images or even to other identities, it cannot be entirely image-specific, but must somehow bias the perception of the other presented identities. As in Experiment 1, there was a shift in the average selection of all participant groups toward the negative pole (see Figure 5D), $M=-2.55$; one sample $t$-test: $t(47)=-2.362$ (one-tailed); $p=.011 ; d=-0.341$. Thus, the total average value across all groups deviated significantly from the anticipated value of zero (see Experiment 1 for further explanation). This implies that, overall, the participants selected images with a lower brightness than expected. Moreover, the adaptation effect observed in this experiment seems to have been similarly large as the effects of the first experiment. Thus, the observed adaptation effect seems to clearly resist an interval of $3 \mathrm{~s}$.

\section{EXPERIMENT 5}

Since the detected adaptation effects seem to be robust, lasting at least several seconds, it was determined in a further experiment whether the effects also persist for several minutes. Therefore, in Experiment 5 , we separated the adaptation and test phases, and the time interval between both phases was increased to five minutes (adopted from the study of Carbon et al., 2007). If adaptation effects still occur after five minutes, it seems plausible that the effects are not just on a sensory level, but that higher cognitive processing mechanisms are involved.

\section{Method}

\section{PARTICIPANTS}

Forty-eight undergraduate students from the Medical School Hamburg ( 32 females, 16 males, $M_{\text {age }}=23.7$ years, range 19 to 32 years) were tested individually. The sample size as well as all study requirements and conditions were the same as in the previous experiments. None of the participants had taken part in the previous experiments.

\section{APPARATUS AND STIMULI}

The apparatus and stimuli were the same as those used in Experiments 1 and 4.

\section{PROCEDURE}

In contrast to the previous experiments, the adaptation and test phases were separated in this experiment. As illustrated in Figure 4, the trials of the adaptation phase started, as in the previous experiments, with a fixation cross (displayed for $500 \mathrm{~ms}$ and placed in the center of the subsequent stimulus position) before presenting the adaptor. Depending on which group they belonged to, participants then either inspected an ORIGINAL image or one of the two extreme versions of this image (MINUS EXTREME or PLUS EXTREME) for 2-4 s. The adaptor was placed in one of six different screen positions. The different parameters (i.e., presentation time and position) were balanced across trials. After the exposure to the adaptor, a backward mask appeared for $150 \mathrm{~ms}$, followed by a screen displaying a gender cover task (saying in German: "Is this person a woman or a man?"). The selection was indicated by pressing the "A" (man) or "L" (woman) keys. As in the previous experiments, the image sets presented in the adaptation phase were balanced across participants. After the adaptation phase, there was a 5 minutes break in which a geographical text was presented to the participants to prevent the previously seen images from being mentally recalled.

Following this break, the two-alternative forced-choice test from the previous experiments was presented. Stimulus position was again randomized and balanced across trials. As in the prior experiments, all three celebrity groups were displayed in the test phase to apply all three transfer levels (pictorial, structural, and cross-identity). The image sets used for the test phase were balanced across participants. The test stimuli were presented for 1.500 ms. Backward masks appeared (for $300 \mathrm{~ms}$ ) after each test-stimulus presentation. Afterwards, participants were asked to select the veridical image from the two previously presented images. The selection was indicated by pressing a specific key ("A" and "L"). The experiment included 360 adaptation and 120 test trials and lasted about 50 minutes. As in all previous experiments, the participants were given a familiarity rating task after the adaptation and test phases.

\section{RESULTS AND DISCUSSION}

On average, $98.6 \%$ of the celebrity faces presented in the experiment were considered to be familiar (individually ranging from $70 \%$ to $100 \%)$. As in the previous experiments, all trials that displayed a ce- 
lebrity that was not recognized by the participant were excluded from further analysis, as well as trials with an RT faster than $200 \mathrm{~ms}$ and the individual outliers (i.e., RT slower than 3 SDs above the mean of each participant). The target selection was used as the dependent variable. The selection was scored according to the alteration of the selected image (a score of -25 was used for MINUS images; 0 for ORIGINAL images; and +25 for PLUS images). In the following results description, effect sizes for nonsignificant values are reported where relevant if $p$ was $<.1$.

A two-way, mixed-design ANOVA was conducted with the between-subjects factor of adaptation group (MINUS EXTREME, ORIGINAL, and PLUS EXTREME) and the within-subjects factor of transfer level (pictorial, structural, and cross-identity). There was no significant main effect of adaptation group, $F(2,45)=30.553, p=.053$, $\mathrm{n}_{\mathrm{p}}{ }^{2}=.122$ (MINUS EXTREME: $M=-6.29, S D=4.82$; ORIGINAL: $M=-4.61, S D=5.67$; PLUS EXTREME: $M=-1.69, S D=5.25$ ). Furthermore, there was no main effect of transfer level, $F(2,90)<1, p$ $=.700$, but there was a significant interaction of adaptation group and transfer level, $F(4,90)=2.803, p=.038, \mathrm{n}_{\mathrm{p}}{ }^{2}=.111$ (see Figure $5 \mathrm{E}$ for an illustration).

Univariate ANOVAs of adaptation group showed a large effect for the pictorial transfer level, $F(2,45)=4.904, p=.012, \mathrm{n}_{\mathrm{p}}{ }^{2}=.179$, but did not show a large effect either for the structural, $F(2,45)=1.451, p$ $=.245$, or cross-identity transfer level, $F(2,45)=3.044, p=.058, \mathrm{n}_{\mathrm{p}}{ }^{2}=$ 119. However, multiple comparisons revealed a significant difference between the MINUS EXTREME and PLUS EXTREME groups for the cross-identity transfer level, $p=.018$. Accordingly, an adaptation effect can be assumed (although mitigated) for both the pictorial and the cross-identity transfer level. Only within the PLUS EXTREME adaptation group, a greater effect was observed for the pictorial compared to the cross-identity transfer level, $p=.042$. Other pairwise comparisons of the transfer levels within the three groups showed no significant differences, $p s>.60$. Thus, the results indicate that faces strongly altered in brightness can bias the subsequent perception of identical images and other identities over a period of at least 5 minutes. As in Experiments 1 and 4, the pictorial transfer level showed the largest effects, indicating an image-specific component within the revealed adaptation effect. However, since there was also an effect for the cross-identity transfer level, the adaptation effect cannot be entirely image-specific, but must somehow affect also higher concepts of faces. As in the previous experiments, there was a shift in the average selection of all participant groups toward the negative pole (see Figure $5 \mathrm{E}$ ), $M=-4.20$; one sample $t$-test: $t(47)=-5.303$ (one-tailed); $p<.001 ; d=-0.765$. Thus, the total average value across all groups deviated significantly from the anticipated value of zero (see Experiment 1 for further explanation). This implies that, overall, the participants selected images with a lower brightness than expected.

\section{GENERAL DISCUSSION}

Studies investigating face adaptation effects can contribute to the understanding of how faces are perceived and how they are mentally rep- resented in memory. However, up to now, the literature on face adaptation has mainly focused on configural face information, neglecting the role of non-configural information such as color or brightness. Thus, the experiments reported here tried to provide an elaborated insight into the role of non-configural face information in the perception and retention of faces.

\section{Existence of Non-configural Adaptation Effects and Their Face Specificity}

The results of the reported experiments indicate that adaptation effects occur for non-configural (i.e., brightness) face information. The exposure to the applied alterations in brightness caused a clear bias (Experiments 1, 4, and eventually 5) in the perception of subsequent faces. Original faces were subsequently perceived as shifted away from the adaptor, while the likelihood increased that slightly manipulated faces (in the direction of the adaptor) would be perceived as the veridical version. Experiments 2 and 3 revealed that the reported effects seem to be rather face-specific. Indeed, we were unable to detect any adaptation effects when using non-face stimuli (unrecognizable, scrambled faces) altered in brightness as adaptors. This suggests that alterations in brightness as such are not able to provoke a shift in the perception of brightness in faces. We interpret this as contextual-dependent face adaptation effects-specifically, that adaptation to a manipulated dimension (e.g., brightness) is linked to the specific context (e.g., faces) in which the manipulation is applied. Thus, adaptation effects on brightness alterations in faces might be provoked only by adaptors showing faces. Experiment 3 used inverted face stimuli as adaptors in order to investigate whether this face-specificity is only superficially available as a certain Gestalt factor of being a face or whether it is based on the expertise-based processing modes of (upright) faces (Schwaninger et al., 2003). As Experiment 3 did not show any adaptation effect, we furthermore agreed on the hypothesis that the adaptation effects found in Experiment 1 are face-specific, or at least specifically face-sensitively operating

Experiments 1, 4, and 5 revealed a shift in the average selection of all participants toward the negative pole. Thus, on average, participants tended to select celebrity faces with a reduced brightness as the original images. This could be caused by a very strong adaptation effect of the MINUS EXTREME adaptation group and/or by a relatively weaker adaptation effect of the PLUS EXTREME adaptation group. Also, a shift of the control (ORIGINAL) group toward the negative range could account for this overall bias toward the negative range. Moreover, the participants in Experiments 2 and 3 also showed an overall bias toward the negative range, although no adaptation effects were revealed in these experiments. Thus, in sum, these results indicate that participants somehow tended to choose images decreased in brightness when asked to select the veridical images. The underlying factors for this bias are not clear yet. It could be that participants perceive brighter images to be slightly manipulated (since, on average, they were not selected as often as darker images). This impression might arise due to the darkened test cabins. The darkening of the test cabins could cause brighter 
TABLE 1.

Overview of the Effects and Effect Sizes of the Individual Studies

\begin{tabular}{rlc}
\hline Experiment 1 & $p$ & Effect size \\
\hline Adaptation group & .001 & $\mathrm{n}_{\mathrm{p}}{ }^{2}=.380$ \\
Pictorial & .001 & $\mathrm{n}_{\mathrm{p}}{ }^{2}=.456$ \\
Structural & .001 & $\mathrm{n}_{\mathrm{p}}{ }^{2}=.320$ \\
Cross-identity & .001 & $\mathrm{n}_{\mathrm{p}}{ }^{2}=.288$ \\
\hline Experiment 2 & & \\
\hline Adaptation group & .730 & - \\
Pictorial & .460 & - \\
Structural & .944 & - \\
Cross-identity & .782 & - \\
\hline Experiment 3 & & \\
\hline Adaptation group & .316 & - \\
Pictorial & .110 & - \\
Structural & .493 & - \\
Cross-identity & .481 & - \\
\hline Experiment 4 & & \\
\hline Adaptation group & .001 & $\mathrm{n}_{\mathrm{p}}{ }^{2}=.393$ \\
Pictorial & .001 & $\mathrm{n}_{\mathrm{p}}{ }^{2}=.477$ \\
Structural & .001 & $\mathrm{n}_{\mathrm{p}}{ }^{2}=.381$ \\
Cross-identity & .001 & $\mathrm{n}_{\mathrm{p}}{ }^{2}=.261$ \\
\hline Experiment 5 & & \\
\hline Adaptation group & .053 & $\mathrm{n}_{\mathrm{p}}{ }^{2}=.112$ \\
Pictorial & .012 & $\mathrm{n}_{\mathrm{p}}{ }^{2}=.179$ \\
Structural & .245 & - \\
Cross-identity & .058 & $\mathrm{n}_{\mathrm{p}}{ }^{2}=.119$ \\
\hline
\end{tabular}

Note. Adaptation group = main effect of the between factor adaptation group (all transfer levels included); pictorial = effect of the between factor adaptation group on the transfer level pictorial; structural $=$ effect of the between factor adaptation group on the transfer level structural; cross-identity = effect of the between factor adaptation group on the transfer level cross-identity. Effects are only shown if the respective $p$ value was below the preset alpha threshold of 05 .

images to be perceived as too bright (this impression should be familiar, e.g., when we reduce the illumination of our cell phone displays in a poorly lit environment), so that participants might have tended to choose darker images as the original image. Another reason could be that an increase in brightness is more obvious as an image manipulation. Hence, participants would clearly identify brighter images as manipulated and would reject them when asked to choose the veridical one. However, the difference between the ORIGINAL image and the slightly decreased version (MINUS) may not be as easy to identify, so that participants might have selected the decreased version in some trials (while rejecting all PLUS versions), resulting in an overall bias into the slightly negative range. Future studies should investigate possible anomalies in the perception of different brightness levels in order to clarify the underlying factors for the bias identified in our experiments.

\section{Processing Levels of Non-configural Adaptation Effects}

Experiment 4 showed that the reported adaptation effects are still present after a delay of $3 \mathrm{~s}$. Experiment 5 also demonstrated effects after a delay of $5 \mathrm{~min}$. Since the brightness of the adaptor stimuli was held constant throughout all experiments, the adaptation effects in Experiment 1 could probably also be considered to be "long-term" (however, boosted by short-term adaptation effects within trials). Thus, adaptation effects on brightness alterations might even last 40 minutes (across the entire experiment) when intensified by trialwise adaptation effects. However, the results of Experiment 5 indicated adaptation effects that are even more robust without any intensification by trialwise adaptation procedures. By implementing a blockwise procedure (i.e., separated adaptation and test phase), Experiment 5 revealed adaptation effects up to 50 minutes. These results indicate that the adaptation effects are not just on a sensory basis or based on simple iconic traces (see, Carbon et al., 2007), but that they affect at least the short-term memory or might even involve long-term memory components (Atkinson \& Shiffrin, 1971; Peterson \& Peterson, 1959; Sperling, 1960). Also, recency effects cannot be considered as the decisive factor for the observed adaptation effects. Previous studies were able to demonstrate that distracting tasks eliminate the recency effects (Glanzer \& Cunitz, 1966). Thus, at least in Experiment 5, recency effects could not be effective since in this experiment, a distractor task was introduced that required the reading of a geographical text. Furthermore, the effects on the cross-identity level (and to some extend also on the structural level) rather contradict a recency effect, since the adaptation and test stimuli differed tremendously.

Based on these results on facial brightness adaptation, it seems that brightness probably is part of the facial representation in memory, and thus of the mental face space. By adapting to brightness alterations, the manipulations are integrated into the mental representation or mental norm of the presented celebrities. This integration mechanism causes a shift of the representations along the brightness dimension toward the adaptor (e.g., after seeing the extremely decreased brightness version, the mental representation shifts along the continuum representing the brightness dimension toward the more decreased brightness pole). Subsequently, stimuli that are more similar to the adaptor are then perceived as more normal or natural, so that participants tend to choose the slightly manipulated versions as the veridical images. Stimuli that are similar to the initial mental representation (i.e., the ORIGINAL image) appear as being slightly manipulated in the opposite direction to the adaptor (Mueller et al., 2020).

Storing brightness alterations in facial memory might not appear very plausible at first glance. Since brightness information is very variable and transient (e.g., changes in illumination alter the brightness of a face), it does not seem to be a stable and characteristic property of a face. Moreover, brightness alterations caused by illumination are often perceptually deducted in order to decode the basic and characteristic color of a persons' face. However, the basic color of a face appears to be a relevant information to store in memory because it can provide valuable information about, for example, an individual's state of health or their ethnic background. In our adaptation paradigm, we altered the brightness of faces independent of the context. Thus, it could be assumed that the alterations in brightness are perceived as inherent in the face when taking into account the unchanging context conditions. This could explain a longer-term retention of brightness alterations. 
Future studies could investigate this issue even further by altering the brightness of the entire image and thus also of the context. Moreover, an extension of the time interval between the adaptation and test phases could clarify whether brightness information is stored even for a longer period of time than just 50 minutes (see e.g., Carbon \& Ditye, 2011; Carbon \& Leder, 2006; Carbon et al., 2007).

An extension of the time interval could also reveal whether the effects are of a temporary or permanent nature. Experiment 5 indicated that the adaptation effects might not be very permanent, since they seem to have been attenuated compared to Experiment 1 (see also Table 1). This might reflect some kind of "resetting" mechanism, which can also be observed in adaptation studies applying configural alterations (see e.g., Carbon \& Ditye, 2011; Carbon \& Leder, 2005; Carbon et al., 2007; Strobach et al., 2011). There are two explanations for this decay of effect. It could either occur as an automatic (passive) process due to the robustness of the original representation of a face (i.e., the preadaptation version). Alternatively, the delay between the adaptation and test phases might provide an opportunity for a re-encounter with the original image (whether mental or real). This could then lead to a readaptation mechanism back to the original (preadaptation) image (see e.g., Carbon \& Ditye, 2011; Mueller et al., 2020). To the authors' knowledge, the nature of the recalibration process is not yet fully understood. Hence, future studies systematically investigating the link between temporal aspects and recalibration mechanisms would be of great benefit. Moreover, future studies should also compare the robustness of adaptation effects on configural versus non-configural face information. Studies of this kind could reveal possible differences in the valence of facial information for face perception and/ or representation. It could be possible, for instance, that adaptation to color alterations is less robust than adaptation to configural face information since color alterations are naturally very transient (e.g., due to different lightning conditions). Thus, it could be assumed that more transient information would probably not be processed on a very high level and hence would also have a less important role in storing (for a discussion on the valence of different face information see e.g., Mueller et al., 2020; Yamashita et al., 2005).

Experiments 1, 4, and 5 indicated not only a general adaptation effect, but also revealed different transfer effects. For Experiments 1 and 4, adaptation effects could be demonstrated for all transfer levels (pictorial, structural, and cross-identity). The results revealed a specific pattern: The pictorial transfer level shows numerically a larger effect compared to the structural and the cross-identity levels (see Table 1). This pattern could also be found in adaptation studies altering configural face information (e.g., Carbon \& Ditye, 2011; Strobach et al., 2011). Although the adaptation effect seems to transfer across images of the same and different identities, there must be an image-specific component within this effect. Thus, when presenting images that differ from the adaptor, the image-specific component would be absent, leading to a lower effect on the structural and cross-identity levels.

The results on the structural level indicate that adaptation transfers well across different images of the same identity. Thus, the adaptation effects are not just image-specific, but must also have some kind of identity-specific component. Furthermore, these results support the view that the face representations stored in memory must be quite flexible, since adaptation occurs despite great differences between the stimuli (i.e., adaptation and test stimuli). The facial representations must either contain a large variety of facial information or a basic and minimalistic facial structure, allowing a face to be recognized despite various changes. However, the nature of facial representations is not yet clear. Since adaptation effects occur by altering very diverse face information, a face representation containing a large variety of information seems to be more likely (see, Mueller et al., 2020).

While the results on the structural transfer level support the assumption that the observed adaptation effects are somehow identityspecific, the results on the cross-identity level indicate that the effects also transfer between identities. This suggests some kind of hierarchical processing where adaptation affects not only a specific face representation, but also superordinate category representations (e.g., prototype representations of different ethnicities, genders, etc.) or a generic face norm (i.e., a prototype for faces in general) that also leads to alterations in other face representations belonging to a given subpopulation. Hence, adaptation must somehow alter underlying face structures, common face information, or face dimensions, leading to an adaptation effect in faces other than the observed one (Carbon \& Ditye, 2011; Jaquet \& Rhodes, 2008; Jaquet, Rhodes, \& Hayward, 2008; Rhodes et al., 2005). With regard to the results of Experiments 2 (i.e., no adaptation effect when presenting scrambled faces) and 3 (i.e., no adaptation effect when presenting inverted faces), the adaptation effects on the different transfer levels clearly underline the face specificity of the observed effects. For example, adaptation effects occur across identical images (pictorial level) and despite extreme differences of the adaptation and test stimuli (structural and cross-identity levels) as long as the images display faces. However, identical images also showing faces but being rotated by $180^{\circ}$ do not provoke adaptation effects because the face processing is disrupted.

While in Experiments 1 and 4, adaptation effects could be observed for all transfer levels, adaptation effects in Experiment 5 only occurred on a pictorial and (probably) on a cross-identity level (see Table 1). It remains unclear why a structural transfer did not occur. It is not reasonable that the adaptation has an impact on the generic face norm or a category representation (e.g., gender), but not on the representation of the observed identity. If a superordinate representation is altered through adaptation, then the representation of the specific identity belonging to this superordinate category should be altered, too. A more detailed analysis of the data revealed an irregularity within the MINUS EXTREME adaptation group. From a descriptive perspective, one participant of this group scored very high compared to the mean of the group $\left(M_{\text {group }}=-6.28 ; M_{\text {participant }}=+5.98\right)$ and was the only one showing a value within the positive range. When excluding the participant from the data analysis, an effect for the main factor of adaptation group was revealed which was absent before, $F(2,45)=4.622 ; p=.015 ; \mathrm{n}_{\mathrm{p}}{ }^{2}=$ .174. When analyzing the interaction, $F(4,42)=2.528 ; p=.050 ; \mathrm{n}_{\mathrm{p}}{ }^{2}=$ .103 , more specific, univariate ANOVAs of the factor adaptation group showed an effect of the pictorial, $F(2,45)=6.639, p=.003, \mathrm{n}_{\mathrm{p}}{ }^{2}=.232$, 
and cross-identity transfer levels, $F(2,45)=4.501, p=.017, \mathrm{n}_{\mathrm{p}}{ }^{2}=.170$, but no effect for the structural transfer level, $F(2,45)=2.242, p=.118$. However, multiple comparisons also revealed an adaptation effect on the structural level between the MINUS EXTREME and PLUS EXTREME adaptation groups, $p=.046$. Thus, the non-representative response behavior of the participants might account for the missing adaptation effect on the structural transfer level. However, other reasons cannot be identified by the authors.

The reported experiments clearly show that adaptation also occurs for non-configural face alterations, here operationalized by brightness. The effects probably operate not just on a sensory level, but also affect representational memory. While previous studies investigating nonconfigural face information mainly emphasized the role of this type of face information in face perception (e.g., Lee \& Perrett, 1997; Liu et al., 2005; Rakover \& Teucher, 1997; Yamashita et al., 2005), the reported experiments clearly demonstrate that non-configural face information is also stored (probably on a long-term basis) in memory. Thus, nonconfigural face information most probably plays a significant role in face identification. The constant update of the information enclosed in the mental representations stored in memory probably facilitates the recognition and differentiation of specific faces from other faces. However, an updating of superordinate category representations or generic norms (i.e., on the cross-identity level) most likely results in a more accurate prototype of specific face categories (e.g., ethnicity, gender, etc.), also leading to a better and more efficient differentiation of different face categories and, thus, faces in general. Being equipped with such an ability to adapt our sensory system provides a stable perception of the faces surrounding us despite the ongoing variations to which they are exposed.

\section{Conclusion}

We think that the reported experiments give a deeper insight into the theoretical understanding of face perception and the mental representation of faces. While previous studies on non-configural face information were able to demonstrate the relevance of non-configural face information in face perception, our adaptation study also revealed the role of non-configural information in the retention of faces. This was done by addressing adaptation effects on brightness alterations. The reported experiments clearly show very robust face-specific adaptation effects on brightness alterations that probably alter the mental face representations enduringly. Thus, brightness information, although very transient in a natural context, seems to be stored in memory and is most probably involved when identifying familiar faces. However, future studies should investigate even longer time ranges between the adaptation and test phases in order to determine whether adaptation effects on nonconfigural face information is as robust and sustainable as adaptation effects on configural face information. This also includes adaptation studies on further non-configural face information in order to understand the qualities and amounts of information responsible for fast, flexible and accurate recognition of faces. This might help to define which face information is generally stored in memory and whether these different types of information have the same valence and longevity in storage.

\section{ACKNOWLEDGEMENTS}

This research was kindly supported by a grant to ClausChristian Carbon and Tilo Strobach from the Deutsche Forschungsgemeinschaft (DFG, CA 917/7-1; STR 1223/4-1). We would like to thank Kim Sievers, Salman Wakilzada, Romy Staedtler and Maximilian Kischkat for their assistance in data collection.

Parts of the data reported here were presented as preliminary data at the following conferences: Annual Meeting of the Psychonomic Society (2018, New Orleans; 2019, Montréal), Annual Meeting of Experimental Psychologists (2019, London), European Conference on Visual Perception (2019, Leuven), Conference of the European Society for Cognitive Psychology (2019, Tenerife)

\section{FOOTNOTES}

${ }^{1}$ Musicians (Adele, Amy Winehouse, Ed Sheeran, Justin Bieber, Justin Timberlake, Katy Perry, Pink, Rihanna, Selena Gomez), actors (Cameron Diaz, Daniel Radcliffe, Elyas M'Barek, Emma Watson, George Clooney, Jennifer Aniston, Leonardo DiCaprio, Til Schweiger, Will Smith), politicians (Angela Merkel, Barack Obama, Donald Trump, Queen Elizabeth, Vladimir Putin), sport celebrities (Boris Becker, Cristiano Ronaldo, Joachim Löw), models (Heidi Klum, Paris Hilton) and entertainers (Thomas Gottschalk, Dieter Bohlen).

2 Thus, the greatest possible adaptation effect toward a reduced brightness would be reflected by always selecting a MINUS image (score of -25) in trials displaying a MINUS image and an ORIGINAL in the test phase and an ORIGINAL image (score of 0 ) in trials displaying an ORIGINAL and a PLUS image in the test phase. The greatest possible adaptation effect toward an increased brightness would be reflected by always selecting a PLUS image (score of +25$)$ in trials displaying a PLUS image and an ORIGINAL in the test phase and an ORIGINAL image (score of 0 ) in trials displaying an ORIGINAL and a MINUS image in the test phase. Since the test trial versions are balanced across the experiment, the dependent variable can range from -12.5 to +12.5 maximum.

\section{REFERENCES}

Atkinson, R. C., \& Shiffrin, R. M. (1971). The control processes of shortterm memory. Retrieved from Stanford, California.

Bach, M. (1996). The Freiburg Visual Acuity Test - Automatic measurement of visual acuity. Optometry and Vision Science, 73(1), 49-53. doi:10.1097/00006324-199601000-00008 المالسلس

Cabeza, R., \& Kato, T. (2000). Features are also important: Contributions of featural and configural processing to face recognition. Psychological Science, 11(5), 429-433. doi:10.1111/14679280.00283 المالسليل

Carbon, C. C., \& Ditye, T. (2011). Sustained effects of adaptation on the perception of familiar faces. Journal of Experimental Psychology: Human Perception and Performance, 37(3), 615-625. doi:10.1037/ 


\section{a0019949}

Carbon, C. C., \& Ditye, T. (2012). Face adaptation effects show strong and long-lasting transfer from lab to more ecological contexts. Frontiers in Psychology, 3(2), 1-6. doi:10.3389/fpsyg.2012.00003 سلسلس Carbon, C. C., \& Leder, H. (2005). Face adaptation: Changing stable representations of familiar faces within minutes? Advances in Cognitive Psychology, 1(1), 1-7. doi:10.2478/v10053-008-0038-8 الس السلسل

Carbon, C. C., \& Leder, H. (2006). The Mona Lisa effect: Is 'our' Lisa fame or fake? Perception, 35(3), 411-414. doi:10.1068/p5452 البلسلسل

Carbon, C. C., Strobach, T., Langton, S. R. H., Harsányi, G., Leder, H., \& Kovács, G. (2007). Adaptation effects of highly familiar faces: Immediate and long lasting. Memory \& Cognition, 35(8), 19661976. doi:10.3758/bf03192929 الس الس الس الس

Clifford, C. W. G. (2002). Perceptial adaptation: motion parallels orientation. Trends in Cognitive Sciences, 6, 136-143. doi:10.1016/ S1364-6613(00)01856-8 سلس

Cohen, J. (1988). Statistical power analysis for the behavioral sciences (2nd ed.). Hillsdale, NJ: Lawrence Erlbaum Associates.

Faul, F., Erdfelder, E., Lang, A.-G., \& Buchner, A. (2007). GPower 3: A flexible statistical power analysis program for the social, behavioral, and biomedical sciences. Behavior Research Methods, 39, 175-191. doi:10.3758/BF03193146 سلس

Fox, C. J., \& Barton, J. J. S. (2007). What is adapted in face adaptation? The neural representations of expression in the human visual system. Brain Research, 1127(1), 80-89. doi:10.1016/j.brainres.2006.09.104 سلس سلس

Ghuman, A. S., McDaniel, J. R., \& Martin, A. (2010). Face adaptation without a face. Current Biology, 20(1), 32-36. doi:10.1016/j. cub.2009.10.077 سلس

Gibson, J. J. (1933). Adaptation, after-effect and contrast in the perception of curved lines. Journal of Experimental Psychology, 16, 1-31. doi:10.1037/h0074626

Glanzer, M., \& Cunitz, A. R. (1966). Two storage mechanisms in free recall. Journal of Verbal Learning and Verbal Behaviour, 5(4), 351-360. doi:10.1016/S0022-5371(66)80044-0 0

Ishihara, S. (1917). Tests for Colour Blindness. Tokyo: Handaya Hongo Harukuch.

Jaquet, E., \& Rhodes, G. (2008). Face aftereffects indicate dissociable, but not distinct, coding of male and female faces. Journal of Experimental Psychology: Human Perception and Performance, 34(1), 101-112. doi:10.1037/0096-1523.34.1.101

Jaquet, E., Rhodes, G., \& Hayward, W. G. (2008). Race-contingent aftereffects suggest distinct perceptual norms for different race faces. Visual Cognition, 16(6), 734-753. doi:10.1080/13506280701350647 المالسلسلس

Köhler, W., \& Wallach, H. (1944). Figural aftereffects: An investigation of visual processes. Proceedings of the American Philosophical Society, 88, 269-357. السلسلس

Lai, M., Oruc, I., \& Barton, J. J. (2012). Facial age after-effects show partial identity invariance and transfer from hands to faces. Cortex, 48(4), 477-486. doi:10.1016/j.cortex.2010.11.014 السلسلسلس

Lee, K. J., \& Perrett, D. (1997). Presentation-time measures of the effects of manipulations in colour space on discrimination of famous faces. Perception, 26(6), 733-752. doi:10.1068/p260733 السلسلس

Leopold, D. A., O'Toole, A. J., Vetter, T., \& Blanz, V. (2001). Prototypereferenced shape encoding revealed by high-level aftereffects. Nature Neuroscience, 4(1), 89-94. doi:10.1038/82947 السلسلسلس

Liu, C. H., Collin, C. A., Farivar, R., \& Chaudhuri, A. (2005). Recognizing faces defined by texture gradients. Perception \& Psychophysics, 67(1), 158-167. doi:10.3758/BF03195019 السلسلس

Macho, S., \& Leder, H. (1998). Your eyes only? A test of interactive influence in the processing of facial features. Journal of Experimental Psychology: Human Perception and Performance, 24(5), 1486-1500. doi:10.1037/0096-1523.24.5.1486 سلس

Maurer, D., Le Grand, R., \& Mondloch, C. J. (2002). The many faces of configural processing. Trends in Cognitive Sciences, 6(6), 255-260. doi:10.1016/S1364-6613(02)01903-4 لس

Meinhardt-Injac, B., Persike, M., \& Meinhardt, G. (2013). Holistic face processing is induced by shape and texture. Perception, 42(7),

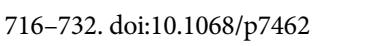

Mondloch, C. J., Le Grand, R., \& Maurer, D. (2002). Configural face processing develops more slowly than featural face processing. Perception, 31(5), 553-566. doi:10.1068/p3339 المالسلسلس

Mueller, R., Utz, S., Carbon, C. C., \& Strobach, T. (2020). Face adaptation and face priming as tools for getting insights into the quality of face space. Frontiers in Psychology, 11(166), 1-17. doi:10.3389/ fpsyg.2020.00166 السلمالسلس

Nestor, A., \& Tarr, M. J. (2008). Gender recognition of human faces using color. Psychological Science, 19(12), 1242-1246. doi:10.1111/ j.1467-9280.2008.02232.x

O'Neil, S. F., \& Webster, M. A. (2011). Adaptation and the perception of facial age. Visual Cognition, 19(4), 534-550. doi:10.1080/135062 85.2011 .561262 سلس الس الس

Otten, M., \& Banaji, M. R. (2012). Social categories shape the neural representation of emotion: Evidence from a visual face adaptation task. Frontiers in Integrative Neuroscience, 6, 9. doi:10.3389/ fnint.2012.00009 سلب

Peterson, L., \& Peterson, M. J. (1959). Short-term retention of individual verbal items. Journal of Experimental Psychology, 58(3), 193-198. doi:https://doi.org/10.1037/h0049234

Rakover, S. S., \& Teucher, B. (1997). Facial inversion effects: Parts and whole relationship. Perception \& Psychophysics, 59(5), 752-761. doi:10.3758/BF03206021 سلس

Rhodes, G., Robbins, R., Jaquet, E., McKone, E., Jeffery, L., \& Clifford, C. W. G. (2005). Adaptation and face perception - How aftereffects implicate norm-based coding of faces. In C. W. G. Clifford \& G. Rhodes (Eds.), Fitting the mind to the world - Adaptation and after-effects in high-level vision (1st ed., pp. 213-240). Oxford University Press.

Schwaninger, A., Carbon, C. C., \& Leder, H. (2003). Expert face processing: Specialization and constraints. In G. Schwarzer \& H. Leder (Eds.), The development of fac processing (pp. 81-97). Göttingen: Hogrefe \& Huber.

Sperling, G. (1960). The information available in brief visual presentations. Psychological Monographs: General and Applied, 74(11), 1-29. doi:10.1037/h0093759 الس الس 
Strobach, T., \& Carbon, C. C. (2013). Face adaptation effects: reviewing the impact of adapting information, time, and transfer. Frontiers in

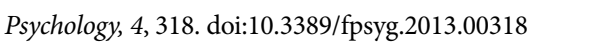

Strobach, T., Ditye, T., \& Carbon, C.-C. (2011). Long-term adaptation effects of highly familiar faces are modulated by adaptation duration. Perception, 40(8), 1000-1004. doi:10.1068/p6986 المبلسلس

Suzuki, S. (2005). High-level pattern coding revealed by brief shape aftereffects. In C. W. G. Clifford \& G. Rhodes (Eds.), Fitting the mind to the world: Adaptation and after-effects in high-level vision (pp. 135-172). Oxford University Press Inc.

Tan, L., \& Ward, G. (2000). A recency-based account of the primacy effect in free recall. Journal of Experimental Psychology, 26(6), 1589-1625. doi:10.1037//0278-7393.26.6.1589 المالس الس الس

Turvey, M. T. (1973). On peripheral and central processes in vision: Inferences from an information-processing analysis of masking with patterned stimuli. Psychological Review, 80(1), 1-52. doi:10.1037/

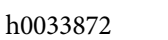

Valentine, T. (1988). Upside-down faces: A review of the effect of inversion upon face recognition. British Journal of Psychology, 79(4),

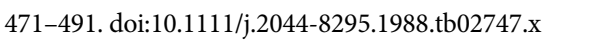

Valentine, T. (1991). A unified account of the effects of distinctiveness, inversion, and race in face recognition. The Quarterly Journal of Experimental Psychology Section A, 43(2), 161-204. doi:10.1080/14640749108400966 المالسلم
Valentine, T., Lewis, M. B., \& Hills, P. J. (2016). Face-space: A unifying concept in face recognition research. The Quarterly Journal of Experimental Psychology, 69(10), 1996-2019. doi:10.1080/1747021 8.2014 .990392 سلس

Webster, M. A., Kaping, D., Mizokami, Y., \& Duhamel, P. (2004). Adaptation to natural facial categories. Nature, 428, 557-561. doi:10.1038/nature02420 الم

Webster, M. A., \& MacLeod, D. I. (2011). Visual adaptation and face perception. Philosophical transactions of the Royal Society of London. Series B, Biological Sciences, 366(1571), 1702-1725. doi:10.1098/

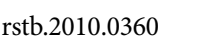

Webster, M. A., \& MacLin, O. H. (1999). Figural aftereffects in the perception of faces. Psychonomic Bulletin \& Review, 6(4), 647-653. doi:10.3758/BF03212974 المبلسلس

Yamashita, J. A., Hardy, J. L., De Valois, K. K., \& Webster, M. A. (2005). Stimulus selectivity of figural aftereffects for faces. Journal of Experimental Psychology: Human Perception and Performance, 31(3), 420-437. doi:10.1037/0096-1523.31.3.420 الفالسلسل|

Yin, R. K. (1969). Looking at upside-down faces. Journal of Experimental Psychology, 81(1), 141-145. doi:10.1037/h0027474 المالسلكال

RECEIVED 18.05.2020 | ACCEPTED 27.04.2021 